University of Montana

ScholarWorks at University of Montana

2012

\title{
Some Explicit Solutions for a Class of One-Phase Stefan Problems
}

Olawanle P. Layeni

University of Cape Town, Rondebosch, South Africa

Jesse V. Johnson

University of Montana - Missoula, jesse.johnson@mso.umt.edu

Follow this and additional works at: https://scholarworks.umt.edu/cs_pubs

Part of the Computer Sciences Commons

Let us know how access to this document benefits you.

\section{Recommended Citation}

Layeni, Olawanle P. and Johnson, Jesse V., "Some Explicit Solutions for a Class of One-Phase Stefan Problems" (2012). Computer Science Faculty Publications. 23.

https://scholarworks.umt.edu/cs_pubs/23

This Conference Proceeding is brought to you for free and open access by the Computer Science at ScholarWorks at University of Montana. It has been accepted for inclusion in Computer Science Faculty Publications by an authorized administrator of ScholarWorks at University of Montana. For more information, please contact

scholarworks@mso.umt.edu. 


\title{
Some Explicit Solutions for a Class of One-Phase Stefan Problems
}

\author{
Olawanle P. Layeni* and Jesse V. Johnson ${ }^{\dagger}$ \\ ${ }^{*}$ Center for Research in Computational and Applied Mechanics, University of Cape Town, 7701 Rondebosch, \\ South Africa \\ † Department of Computer Science, University of Montana, Missoula 59812, U.S.A.
}

\begin{abstract}
Salva and Tarzia, [N.N. Salva, D.A. Tarzia, J. Math. Anal. Appl. 379 (2011) 240 - 244], gave explicit solutions of a similarity type for a class of free boundary problem for a semi-infinite material. In this paper, through an elementary approach and less stringent assumption on data, we obtain more general results than those given by their central result, and thereby construct explicit solutions for a wider class of Stefan problems with a type of variable heat flux boundary conditions. Further, explicit solutions of certain forced one-phase Stefan problems are given.
\end{abstract}

Keywords: Explicit solution, Stefan problem, differential-difference equations

PACS: $44.05 .+\mathrm{e}$

\section{INTRODUCTION}

In this paper we give some explicit solutions to a class of one-phase Stefan problem driven by the partial differential equation $(P D E)$

$$
\frac{\partial u}{\partial t}-\frac{\partial^{2} u}{\partial x^{2}}=\gamma \mathfrak{H}(x, t), \quad x \in[0, \delta(t)), \quad t>0
$$

with dimensionless spatial and temporal variables, respectively, $x \in \mathbb{R}$ and $t \in \mathbb{R}_{0}^{+} \stackrel{\text { def }}{=} \mathbb{R}^{+} \cup\{0\}$, and a moving front defined through a continuously differentiable $\delta_{2}$, with nice enough forcing term $\gamma \mathfrak{H}(\delta(t), t), \gamma$ real, satisfying initial and boundary values of the form

$$
\begin{aligned}
\left.u\right|_{t=0}=e_{0} x^{p},\left.u\right|_{x=0} & =e_{1} \delta_{2}^{p}(t),\left.\frac{\partial u}{\partial x}\right|_{x=0}=e_{2} \delta_{2}^{p}(t) \frac{d \delta(t)}{d t} \\
\left.u\right|_{x=\delta(t)} & =e_{4} \delta(t)+e_{3} \delta_{2}^{p}(t),\left.\frac{\partial u}{\partial x}\right|_{x=\delta(t)}=e_{4}+e_{5} \delta^{p}(t) \frac{d \delta(t)}{d t}
\end{aligned}
$$

where $p, e_{l} \in \mathbb{R}$.

Eqs. (1) - (2) is a class which contains the classical Stefan melting problem $\left(\gamma=0, p=0, e_{1}=1, e_{2} \geq 0, e_{3}=0\right.$, $\left.e_{4}=0\right)$; Huppert's model [2] for hot turbulent flow over a cold surface $\left(\gamma=0, p=0, e_{4} \neq 0\right)$ if the interval in Eq. (1) is replaced with $(\delta(t),+\infty)$; Salva and Tarzia [1] $\left(\gamma=0, p=1, e_{4}=0\right)$, to mention a few.

Several authors, for instance $[1,3,4,5]$, have obtained explicit solutions for one and multi-phase Stefan problems. A favored approach in the literature is a reduction of Eq. (1) to an ordinary differential equation $(O D E)$ in a similarity variable proportional to $x / \sqrt{t}$.

Starting from such an elementary approach, which evolves into the study of an equivalent class of differentialdifference equation $(D D E)$ to Eq. (1), we obtain some pertinent explicit solutions.

\section{MAIN RESULTS}

In this section, we shall start by studying explicit solutions of Eq. (1) in the absence of forcing, and with a more general moving boundary $x \in(\omega(t), \delta(t))$. The approach taken involves that of the suggestion of a solution $u(x, t)$ which takes the form of an infinite series in a similarity variable $\xi \stackrel{\text { def }}{=}(x-\omega(t)) /(\delta(t)-\omega(t))$. Subsequently, a

Numerical Analysis and Applied Mathematics ICNAAM 2012

(C) 2012 American Institute of Physics 978-0-7354-1091-6/\$30.00 
constructive study of a class of exponential forcing term in spatio-temporal variables is given for the first time. Our first result is summarized in the following Lemma.

Lemma 1. The PDE

$$
\frac{\partial u}{\partial t}-\frac{\partial^{2} u}{\partial x^{2}}=0, x \in(\omega(t), \delta(t)),
$$

with real valued $\omega(t)$ and $\delta(t)$, has a similarity-like solution

$$
u(x, t)=\alpha_{-1} x+\sum_{j=0}^{\infty} \alpha_{j}(t) \xi^{j}
$$

if its coefficients $\alpha_{j}(t), \alpha_{-1}$ known constant, are determined by the differential-difference equation

$$
\left\{\begin{array}{l}
\chi^{2}(t) \alpha_{j}^{\prime}(t)-(j+2)(j+1) \alpha_{j+2}(t)+(j+1) \chi(t) \omega^{\prime}(t) \alpha_{j+1}(t)-j \chi(t) \chi^{\prime}(t) \alpha_{j}(t)=0 \\
\chi(t)=\delta(t)-\omega(t)
\end{array}\right.
$$

Moreover, suppose the $\alpha_{j}$ are chosen in a parametrized form such that $\alpha_{j}(t)=\alpha_{j}(t ; p)=a_{j} \delta^{p}(t), \omega(t)=\kappa \delta(t)$, $\chi(0)=0=\delta(0)$, with $\kappa, a_{j} \in \mathbb{R}$. Then,

$$
\begin{aligned}
u(x, t) \equiv & u(x, t ; \kappa, p)=\alpha_{-1} x+\delta^{p}(t)\left[C_{1}+C_{2} \xi+\left(\frac{1}{2} \theta^{2}(-1+\kappa)\left(p(-1+\kappa) C_{1}-\kappa C_{2}\right)\right) \xi^{2}\right. \\
& \left.+\left(\frac{1}{6} \theta^{2}(-1+\kappa)^{2}\left(-p \theta^{2}(-1+\kappa) \kappa C_{1}+\left(-1+p+\theta^{2} \kappa^{2}\right) C_{2}\right)\right) \xi^{3}+\ldots\right]
\end{aligned}
$$

where $C_{1}$ and $C_{2}$ are arbitrary constants.

The proof of Lemma 1, which relies on direct substitution and a systematic solution (albeit not closed) of the pertinent $D E$

$$
\left\{\begin{array}{l}
\theta^{2}(1-\kappa)\left(a_{j+1} \kappa(j+1)+a_{j}(1-\kappa)(p-j)\right)=2 a_{j+2}(j+2)(j+1) \\
\omega_{2}(t)=\theta \sqrt{t}
\end{array}\right.
$$

$p \in \mathbb{R}, \kappa \in(-\infty, 1)$, and $\theta \in \mathbb{R}_{0}^{+}$, is direct. The solution to Eq. (3) in the case wherein $\kappa=0$ and $p=1$, which is the core of the results of [1], has a series expression which can be sieved from equation (6). The next theorem proffers a closed form solution for the more general case where $\kappa=0$ and $p \in \mathbb{R}$.

Theorem 1. Suppose that $\kappa=0$ in Lemma 1. Then for $\gamma=0, P D E$ (1) has a class of solutions, which is parametrized by $p \in \mathbb{R}$ and expressible in terms of Kummer's confluent hypergeometric function ${ }_{1} \mathrm{~F}_{1}$ as

$$
u(x, t)=\alpha_{-1} x+\delta^{p}(t)\left[{ }_{1} \mathrm{~F}_{1}\left(-\frac{p}{2}, \frac{1}{2},-\frac{x^{2} \theta^{2}}{4 \delta^{2}(t)}\right) c_{1}+{ }_{1} \mathrm{~F}_{1}\left(\frac{1-p}{2}, \frac{3}{2},-\frac{x^{2} \theta^{2}}{4 \delta^{2}(t)}\right) \frac{x}{\delta(t)} c_{2}\right],
$$

where $\delta(t)=\theta \sqrt{t}, c_{l}, l \in\{0,1,2\}$ constants.

Furthermore, if initial and boundary value conditions

$$
\left.u\right|_{x=0}=e_{1} \delta_{2}^{p}(t),\left.u\right|_{x=\delta(t)}=e_{4} \delta(t)+e_{3} \delta_{2}^{p}(t),\left.\frac{\partial u}{\partial x}\right|_{x=\delta(t)}=e_{4}+e_{5} \delta^{p}(t) \frac{d \delta(t)}{d t},
$$

are imposed, the relations

$$
\begin{aligned}
& {\left[-\frac{1}{6}(1-p) \theta^{2}{ }_{1} F_{1}\left(\frac{3-p}{2}, \frac{5}{2},-\frac{\theta^{2}}{4}\right)+{ }_{1} F_{1}\left(\frac{1-p}{2}, \frac{3}{2},-\frac{\theta^{2}}{4}\right)\right] c_{2}+\frac{1}{p} \theta^{2}{ }_{1} F_{1}\left(1-\frac{p}{2}, \frac{3}{2},-\frac{\theta^{2}}{4}\right) e_{1}=\theta^{2} \frac{e_{5}}{2},} \\
& { }_{1} F_{1}\left(\frac{1-p}{2}, \frac{3}{2},-\frac{\theta^{2}}{4}\right) c_{2}+{ }_{1} F_{1}\left(\frac{-p}{2}, \frac{1}{2},-\frac{\theta^{2}}{4}\right) e_{1}=e_{3}, \alpha_{-1}=e_{4}
\end{aligned}
$$

hold. 
The case of a non-zero forcing term is studied in a constructive manner. Suppose the forcing term is $\mathfrak{H}(\omega, \delta, x, t)$. In this instance, the series expression corresponding to Eq. (3) upon the assumption of a series expansion (4) is

$$
\begin{aligned}
\sum_{j=0}^{\infty}\left\{\frac{(j+2)(j+1) \alpha_{j+2}(t)}{(\delta(t)-\omega(t))^{2}}-\left[\alpha_{j}^{\prime}(t)-(j+1) \alpha_{j+1}(t) \frac{\delta_{1}^{\prime}}{\delta(t)-\omega(t)}-j \alpha_{j}(t) \frac{(\delta(t)-\omega(t))^{\prime}}{(\delta(t)-\omega(t))}\right]\right\} \\
\quad \times\left(\frac{x-\omega(t)}{\delta(t)-\omega(t)}\right)^{j}=\mathfrak{H}(\omega(t), \delta(t), x, t) .
\end{aligned}
$$

Motivated by the structure of Eq. (11), we shall amongst several possibilities consider a forcing term of the form

$$
\mathfrak{H}(\omega(t), \delta(t), x, t ; m)=m \sum_{j=0}^{\infty} \frac{(j+2)(j+1) a_{j+2}(t)}{(\delta(t)-\omega(t))^{(2-p)}}\left(\frac{x-\omega(t)}{\delta(t)-\omega(t)}\right)^{j}, \quad a_{j}, m \in \mathbb{R},
$$

which induces a system driven by the $D E$

$$
(1-j) \delta(t) \delta^{\prime}(t) a_{j}-(j+2)(j+1)(p-m) a_{j+2}=0 .
$$

In the special case of Eq. (13) where $p \in\{0,1\}, m \neq 0$ and a forcing term of type Eq. (12) imposed, we have the following result.

Theorem 2. The forced one-phase Stefan problem

$$
\frac{\partial^{2} u}{\partial x^{2}}-\frac{\partial u}{\partial t}=\mathfrak{H}_{[p]}, \quad x \in[0, \delta(t))
$$

$p \in\{0,1\}$, such that

$$
\mathfrak{H}_{[p]}= \begin{cases}\frac{-m r_{[0]} \delta(t)}{2 \sqrt{t}(1-m)} \exp \left[-x^{2} /(4(1-m) t)\right] & p=0 \\ \frac{-m r_{[1]} \delta(t)}{2 t(1-m)} \exp \left[-x^{2} /(4(1-m) t)\right] & p=1\end{cases}
$$

$m<1$ or $m>1+\frac{1}{2} \delta(t) \frac{d \delta(t)}{d t}$, has similarity solutions

$$
\begin{aligned}
& \delta(t)=\theta_{[p]} \sqrt{t} \\
& u(x, t)= \\
& \begin{cases}s_{[0]}+\left(r_{[0]} / \theta_{[0]}\right) \sqrt{\pi(1-m)} \operatorname{erf}[x /(2 \sqrt{(1-m) t})] & p=0 \\
s_{[1]} x+\left(r_{[1]} \theta_{[1]} / 2\right) \sqrt{\pi(1-m)^{-1}} x \operatorname{erf}[x /(2 \sqrt{(1-m) t})]+r_{[1]} \theta_{[1]} \sqrt{t} \exp \left[-x^{2} /(4(1-m) t)\right] & p=1\end{cases}
\end{aligned}
$$

where $r, s$ are arbitrary constants.

Moreover, if Eqs. (14), (15) have boundary conditions

$$
\begin{gathered}
\left.u\right|_{x=0}=\varepsilon_{1[p]} \delta^{p}(t),\left.\frac{\partial u}{\partial x}\right|_{x=0}=\varepsilon_{2[p]} \delta^{p}(t) \frac{d \delta(t)}{d t} \\
\left.u\right|_{x=\delta(t)}=\varepsilon_{3[p]} \delta^{p}(t),\left.\frac{\partial u}{\partial x}\right|_{x=\delta(t)}=\varepsilon_{4[p]}+\varepsilon_{5[p]} \delta^{p}(t) \frac{d \delta(t)}{d t}
\end{gathered}
$$

then $s_{[p]}, r_{[p]}$ and $\theta_{[p]}$ are determined from the relations

$$
\left\{\begin{array}{l}
\varepsilon_{1[0]}=s_{[0]}, \varepsilon_{2[0]}=2 r_{[0]} \theta_{[0]}^{2}, \varepsilon_{3[0]}=s_{[0]}+r_{[0]} \sqrt{\pi(m-1)} \operatorname{erf}\left[\theta_{[0]} /(2 \sqrt{1-m})\right] \\
\varepsilon_{4[0]}=0, \varepsilon_{5[0]}=2 r_{[0]} / \theta^{2} \exp \left[\theta_{[0]}^{2} /(4(m-1))\right]
\end{array}\right.
$$


or

$$
\left\{\begin{array}{l}
\varepsilon_{1[1]}=r_{[1]}, \varepsilon_{2[1]}=2 s_{[1]} \theta_{[1]}^{-2}, \\
2 \varepsilon_{1[1]} \exp \left[\theta_{[1]}^{2} /(4(m-1))\right]=\left(2 \varepsilon_{3[1]}-\varepsilon_{4[1]}\right)-\left(\varepsilon_{2[1]}+\varepsilon_{5[1]}\right) \theta_{[1]}^{2} / 2,
\end{array}\right.
$$

according as $p$ is 0 or 1 .

It is easy to see that if $\mathfrak{H}$ is taken such that

$$
\mathfrak{H}(\omega(t), \delta(t), x, t ; m)=\beta \sum_{j=0}^{\infty} \frac{\alpha_{j+1} \omega(t)}{\delta(t)-\omega(t)}\left(\frac{x-\omega(t)}{\delta(t)-\omega(t)}\right)^{j},
$$

our consideration is one which is synonymous to that of the study of a dual moving boundary $P D E$

$$
\frac{\partial u}{\partial t}-\frac{\partial^{2} u}{\partial x^{2}}=\beta \omega(t) \frac{\partial u}{\partial x}, \quad x \in(\omega(t), \delta(t)) .
$$

with the corresponding governing $D D E$

$$
\left\{\begin{array}{l}
\chi^{2}(t) \alpha_{j}^{\prime}(t)-(j+2)(j+1) \alpha_{j+2}(t)+(1-\beta)(j+1) \chi(t) \omega^{\prime}(t) \alpha_{j+1}(t)-j \chi(t) \chi^{\prime}(t) \alpha_{j}(t)=0 \\
\chi(t)=\delta(t)-\omega(t)
\end{array}\right.
$$

In this vein, we give the following Corollary to Theorem 2, one in which $\beta=1, \omega(t)=\kappa \delta(t)$. A special case of this, with $p=0$, was considered by Yi et al. [6] in the study of a one-dimensional free boundary problem in an angular domain.

Corollary 1. The PDE

$$
\frac{\partial u}{\partial t}-\frac{\partial^{2} u}{\partial x^{2}}=\kappa \delta(t) \frac{\partial u}{\partial x}, \quad x \in(\kappa \delta(t), \delta(t))
$$

with boundary conditions Eq. (17) has solutions (16), together with relevant conditions (18) or (19), with

$$
\kappa=1-(1-m)^{-1 / 2}, \quad 1>m \in \mathbb{R} .
$$

\section{CONCLUSION}

In this paper, we have given new explicit solutions to a class of one-phase Stefan problems both in the presence of a spatio-temporal exponential forcing term and in the absence of forcing. Our approach, one which relies on the study of an equivalent differential-difference equation, permitted a generalization of recent and standard results in the literature. Furthermore, this approach naturally admits the square root type evolution of moving fronts and its compatible fields while leaving the opportunity for future consideration of other forms of moving fronts.

\section{REFERENCES}

1. N. N. Salva, D. A. Tarzia, Explicit solution for a Stefan problem with variable latent heat and constant heat flux boundary conditions, J. Math. Anal. Appl. 379 (2011) 240 - 244.

2. H. E. Huppert, Phase changes following the initiation of a hot turbulent flow over a cold solid surface. J. Fluid Mech. 198, (1989) 293 - 319.

3. H. Capart, M. Bellal, D.L. Young, Self-similar evolution of semi-infinite alluvial channels with moving boundaries, J. Sedimentary Res. 77 (2007) 13 - 22.

4. S. Y. J. Lai, H. Capart, Reservoir infill by hyperpycnal deltas over bedrock, Geophys. Res. Lett. 36 (2009) L08402.

5. D. A. Tarzia, An explicit solution for a two-phase unidimensional Stefan problem with a convective boundary condition at the fixed face, in: MAT Ser. A, 8, (2004) 21 - 27.

6. F. Yi, X. Han, An one-dimensional two-phase free boundary problem in an angular domain, Nonlinear Analysis: Real World Applications 8 (3), (2007) 959-979. 
Copyright of AIP Conference Proceedings is the property of American Institute of Physics and its content may not be copied or emailed to multiple sites or posted to a listserv without the copyright holder's express written permission. However, users may print, download, or email articles for individual use. 